\title{
THE ON-LINE IUE UNIFORM LOW DISPERSION ARCHIVE
}

\author{
M. Barylak, W. Wamsteker, C. Driessen, \\ J.R. Munoz, A. Talavera \\ ESA IUE Observatory \\ P.O. Box 54065, 28080 Madrid, Spain
}

\begin{abstract}
After a decade of IUE observations, the dearchiving rate for IUE data in the public domain is more than 1000 images per month, which is more than twice the number of images taken monthly at both groundstations (ie. Goddard Space Flight Center and the ESA Villafrance Satellite Tracking Station). Considering this high interest in IUE data, an effort was made to improve the accessibility of the IUE archive taking into account the availibility of current intercomputer networks.

The result: the IUE Uniform Low Dispersion Archive (ULDA), a further output product (under standard quality control assurance) of the IUE project, supported by an easy to use Support Software Package (USSP). This allows scientists to help themselves to spectra by an interactive search and select procedure followed by a downlink of the data to their own computers for subsequent data reductions.

The underlying concepts of the ULDA together with practical aspects of its installation, distribution and access under the USSP are presented.

Only one month after its distribution in Europe, 4000 spectra have already been chosen from the ULDA, confirming the usefulness of the basic concept.

\section{REFERENCES}

Barylak M., Munoz J.R., Driessen C.: 1987, The Uniform Low Dispersion Archive of the International Ultraviolet Explorer Satellite; Astronomy from Large Databases, ESO Conference and Workshop Proceedings No. 28, pg. 281.

Wamsteker W., Driessen C., Munoz J.R., Hassal B.J., Pasian F., Barylak M., Russo G., Egret D., Murray J., Talavera A.: 1988, IUE-ULDA/USSP: The on-line data archive (low resolution) of the International Ultraviolet Explorer, Astron. Astrophy. Suppl., in preparation.
\end{abstract}

